\title{
MICROFLUIDIC CRYSTALLIZATION OF SURFACTANT- FREE DOPED ZINC SULFIDE NANOPARTICLES FOR OPTICAL BIOIMAGING APPLICATIONS
}

Francesca Tajoli, ${ }^{\mathrm{a}, \mathrm{b}}$ Nicola Dengo,,${ }^{\mathrm{a}, \mathrm{b}}$ Maddalena Mognato, ${ }^{\mathrm{c}}$ Paolo Dolcet, ${ }^{\mathrm{d}}$ Giacomo Lucchini, ${ }^{\mathrm{e}}$ Andrea Faresin, ${ }^{a}$ Jan-Dierk Grunwaldt, ${ }^{\mathrm{d}}$ Xiaohui Huang, ${ }^{\mathrm{f}}$ Denis Badocco, ${ }^{\mathrm{a}}$ Michele Maggini,${ }^{\mathrm{a}, \mathrm{b}}$ Christian Kübel, ${ }^{\mathrm{f}, \mathrm{g}}$ Adolfo Speghini, ${ }^{\text {e* }}$ Tommaso Carofiglio, ${ }^{\mathrm{a}, \mathrm{b} *}$ and Silvia Gross ${ }^{\mathrm{a}, \mathrm{b} *}$

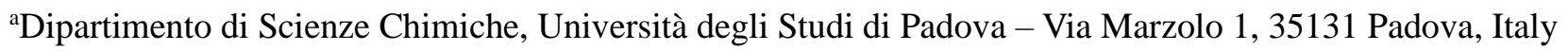
bINSTM, UdR di Padova - Via Marzolo 1, 35131 Padova, Italy

'Dipartimento di Biologia, Università degli Studi di Padova - Via Bassi 58B, 35131 Padova, Italy

${ }^{\mathrm{d} K a r l s r u h e r ~ I n s t i t u t ~ f u ̈ r ~ T e c h n o l o g i e ~(K I T), ~ I n s t i t u t ~ f u ̈ r ~ T e c h n i s c h e ~ C h e m i e ~ u n d ~ P o l y m e r c h e m i e ~(I T C P) ~-~}$ Engesserstr. 20, 76131 Karlsruhe, Germany

${ }^{\mathrm{e}}$ NRG, Dipartimento of Biotecnologie, Università di Verona and INSTM, RU Verona - Strada Le Grazie 15, 37314 Verona, Italy

fKarlsruher Institut für Technologie (KIT), Institut für Nanotechnologie (INT) - Hermann-von-HelmholtzPlatz 1, 76344 Eggenstein-Leopoldshafen, Germany

'Department of Materials \& Earth Sciences, Technical University Darmstadt, Alarich-Weiss-Str. 2, 64287

Darmstadt, Germany

In Supporting Information, the following analyses are reported:

- X-ray Powder Diffraction: fittings of XRPD patterns of undoped and doped ZnS samples (Figures S1, S2, S4, S6, S8, S10) and corresponding refined parameters (Table S1-S6), average crystallite size and lattice constant $a$ as functions of dopant concentrations (Figures S3, S5, S7, S9, S11), XRPD patterns of Nd-, Sm- and Yb-doped ZnS samples compared with undoped one (Figure S12 and S13);

- Transmission Electron Microscopy: HR-TEM micrographs of undoped and Eu-doped ZnS samples (Figure S14 and S17) and TEM micrographs of doped ZnS samples and size distribution histograms (Figure S15, S16, S18-S20);

- X-ray Photoelectroon Spectroscopy: fittings of XPS peaks of undoped and 5 at\% doped ZnS samples (dopants: $\mathrm{Mn}, \mathrm{Eu}, \mathrm{Sm}, \mathrm{Yb}$ ) (Figure S21-S25), surface atomic composition \% of undoped and 5 at\% doped samples (semi-quantitative analysis data) (Table S7), XPS data (binding energies and Auger parameters) of the Sm- and Yb-doped samples (Table S8);

- ICP-MS Analysis: ICP-MS data of the Sm- and Yb-doped samples (Table S9);

- $\quad$ X-ray Absorption spectroscopy: fitted Fourier transformed EXAFS data of Eu- and Nd-doped samples (Figures S26 and S27);

- Absorption and photoluminescence spectroscopy: UV-vis diffuse reflection spectrum of undoped ZnS (Figure S28), picture of undoped and Mn-doped samples under UV illumination (Figure S29), comparison between absorption band of undoped $\mathrm{ZnS}$ and excitation band of Mn-doped samples (Figure S30), photoluminescence spectra of $\mathrm{ZnS}: \mathrm{Eu} 5$ at\% (Figure S31). 
XRPD Diffraction.

Undoped $\mathrm{ZnS}$

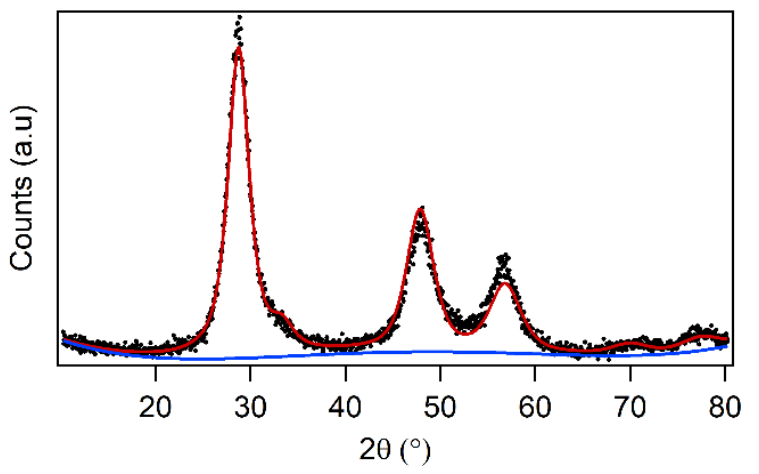

Figure S1. Fitting (red line) of diffractogram of undoped ZnS. Blue line: background.

Table S1. Refined parameters obtained from fitting of diffractogram of undoped $\mathrm{ZnS}$.

\begin{tabular}{ccccccc}
\hline Sample & Crystallite size (nm) & R.m.s. microstrain (\%) & $a(\AA)$ & $\mathrm{R}_{\exp }(\%)$ & $\mathrm{R}_{\mathrm{wp}}(\%)$ & $\chi^{2}$ \\
\hline Undoped $\mathrm{ZnS}$ & $4.6 \pm 0.1$ & $1.5 \pm 0.1$ & $5.37 \pm 0.01$ & 9.58 & 12.76 & 1.77 \\
\hline
\end{tabular}


Mn-doped ZnS
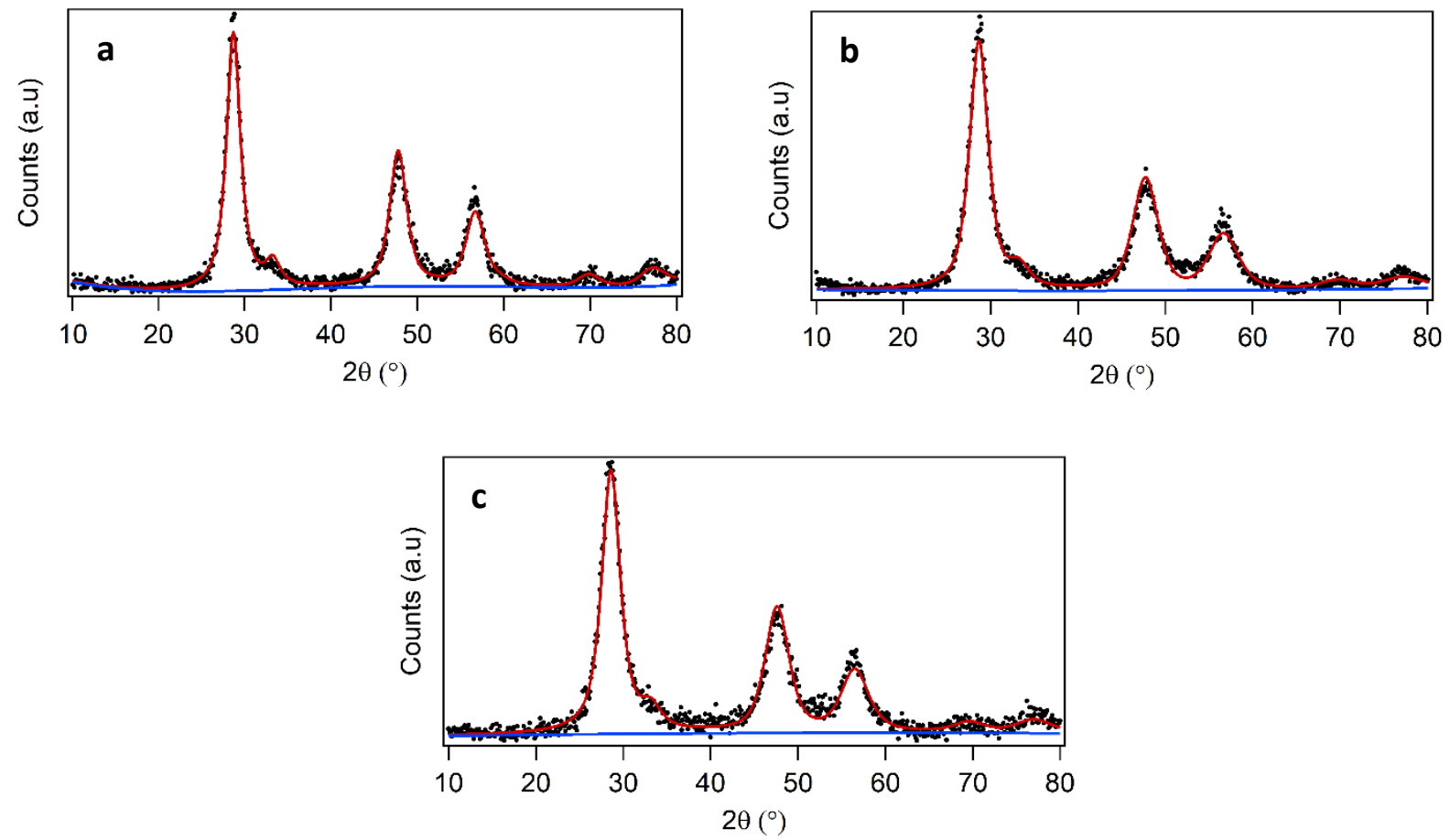

Figure S2. Fitting (red line) of diffractograms of Mn-doped ZnS samples. Doping percentages: 0.1 at\% (a), 1 at $\%$ (b), 5 at $\%$ (c). Blue line: background.

Table S2. Refined parameters obtained from fitting of diffractogram of Mn-doped ZnS.

\begin{tabular}{ccccccc}
\hline Sample & Crystallite size $(\mathrm{nm})$ & R.m.s. microstrain $(\%)$ & $a(\AA)$ & $\mathrm{R}_{\mathrm{exp}}(\%)$ & $\mathrm{R}_{\mathrm{wp}}(\%)$ & $\chi^{2}$ \\
\hline ZnS:Mn 0.1 at\% & $5.9 \pm 0.1$ & $0.8 \pm 0.1$ & $5.38 \pm 0.01$ & 12.13 & 14.14 & 1.36 \\
\hline ZnS Mn 1 at\% & $5.4 \pm 0.2$ & $1.8 \pm 0.1$ & $5.39 \pm 0.01$ & 10.05 & 13.91 & 1.91 \\
\hline ZnS:Mn 5 at\% & $4.8 \pm 0.1$ & $1.4 \pm 0.1$ & $5.40 \pm 0.01$ & 10.10 & 12.37 & 1.50
\end{tabular}
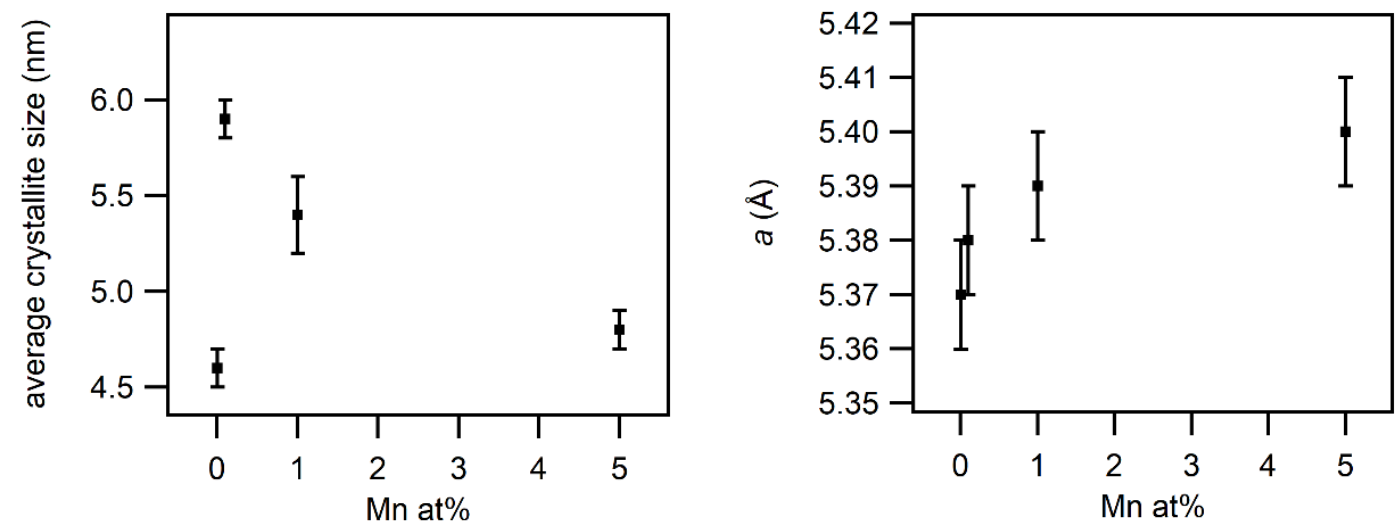

Figure S3. Average crystallite size (left) and lattice constant $a$ (right) obtained from fitting of diffractograms of Mndoped $\mathrm{ZnS}$ as a function of $\mathrm{Mn}$ atomic percentage. 
Eu-doped ZnS

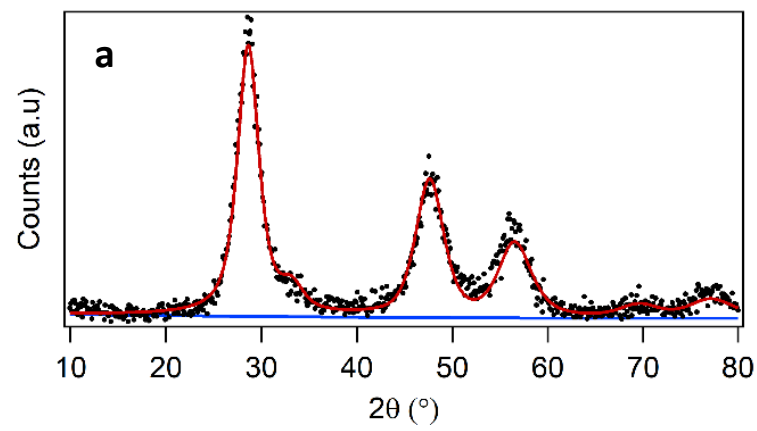

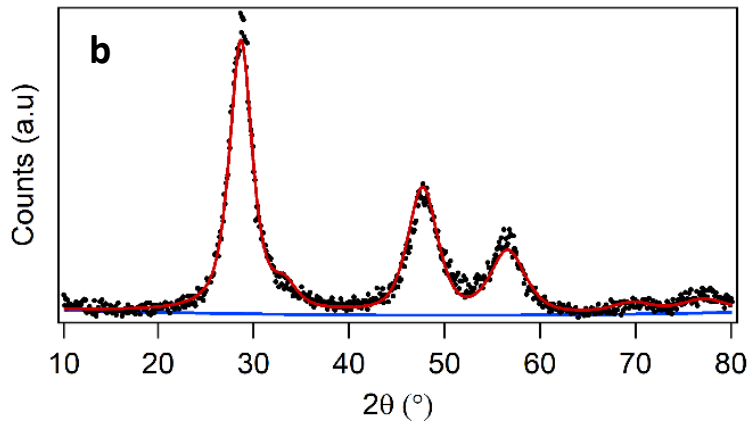

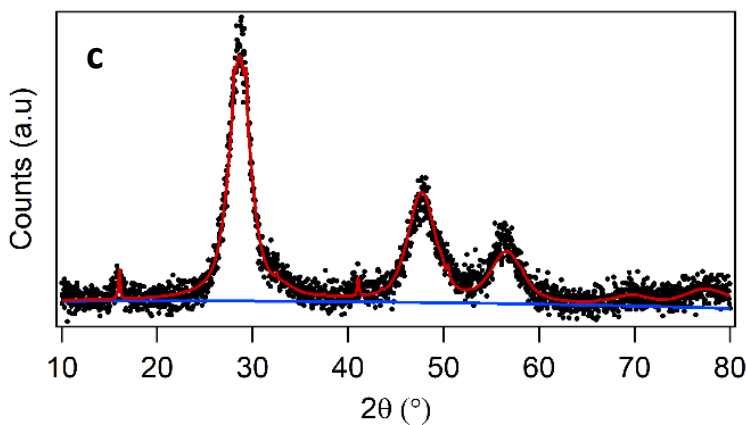

Figure S4. Fitting (red line) of diffractograms of Eu-doped ZnS samples. Doping percentages: 0.1 at $\%$ (a), 1 at $\%$ (b), 5 at $\%$ (c). Blue line: background.

Table S3. Refined parameters obtained from fitting of diffractogram of Eu-doped $\mathrm{ZnS}$.

\begin{tabular}{cccccccc}
\hline Sample & Crystallite size (nm) & R.m.s. microstrain $(\%)$ & $a(\AA)$ & $\mathrm{R}_{\exp }(\%)$ & $\mathrm{R}_{\mathrm{wp}}(\%)$ & $\begin{array}{c}\chi^{2} \\
\mathrm{Eu}(\mathrm{OH})_{3} \\
\text { wt } \%\end{array}$ \\
\hline ZnS:Eu 0.1 at\% & $4.9 \pm 0.2$ & $1.8 \pm 0.1$ & $5.41 \pm 0.01$ & 12.29 & 14.63 & 1.42 & - \\
\hline ZnS:Eu 1 at\% & $4.6 \pm 0.1$ & $1.8 \pm 0.1$ & $5.39 \pm 0.01$ & 9.06 & 11.72 & 1.68 & - \\
\hline ZnS:Eu 5 at\% & $5.1 \pm 0.2$ & $1.7 \pm 0.1$ & $5.39 \pm 0.01$ & 10.50 & 11.32 & 1.16 & $1.8 \pm 0.2$ \\
\hline
\end{tabular}
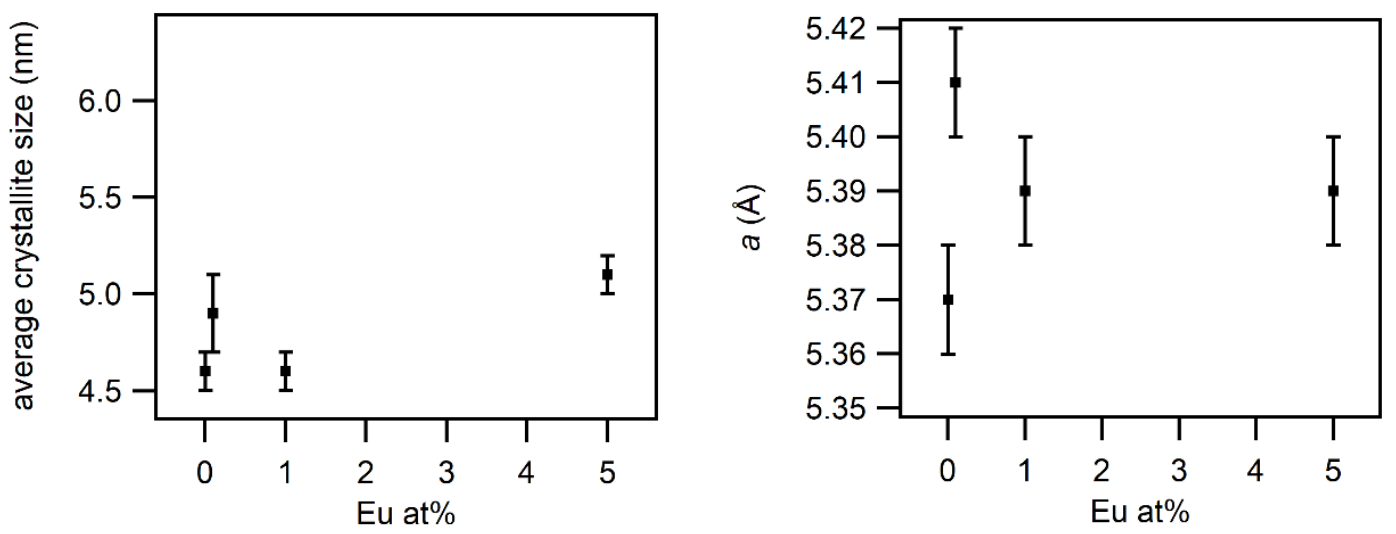

Figure S5. Average crystallite size (left) and lattice constant $a$ (right) obtained from fitting of diffractograms of Eudoped $\mathrm{ZnS}$ as a function of $\mathrm{Eu}$ atomic percentage. 
Nd-doped ZnS
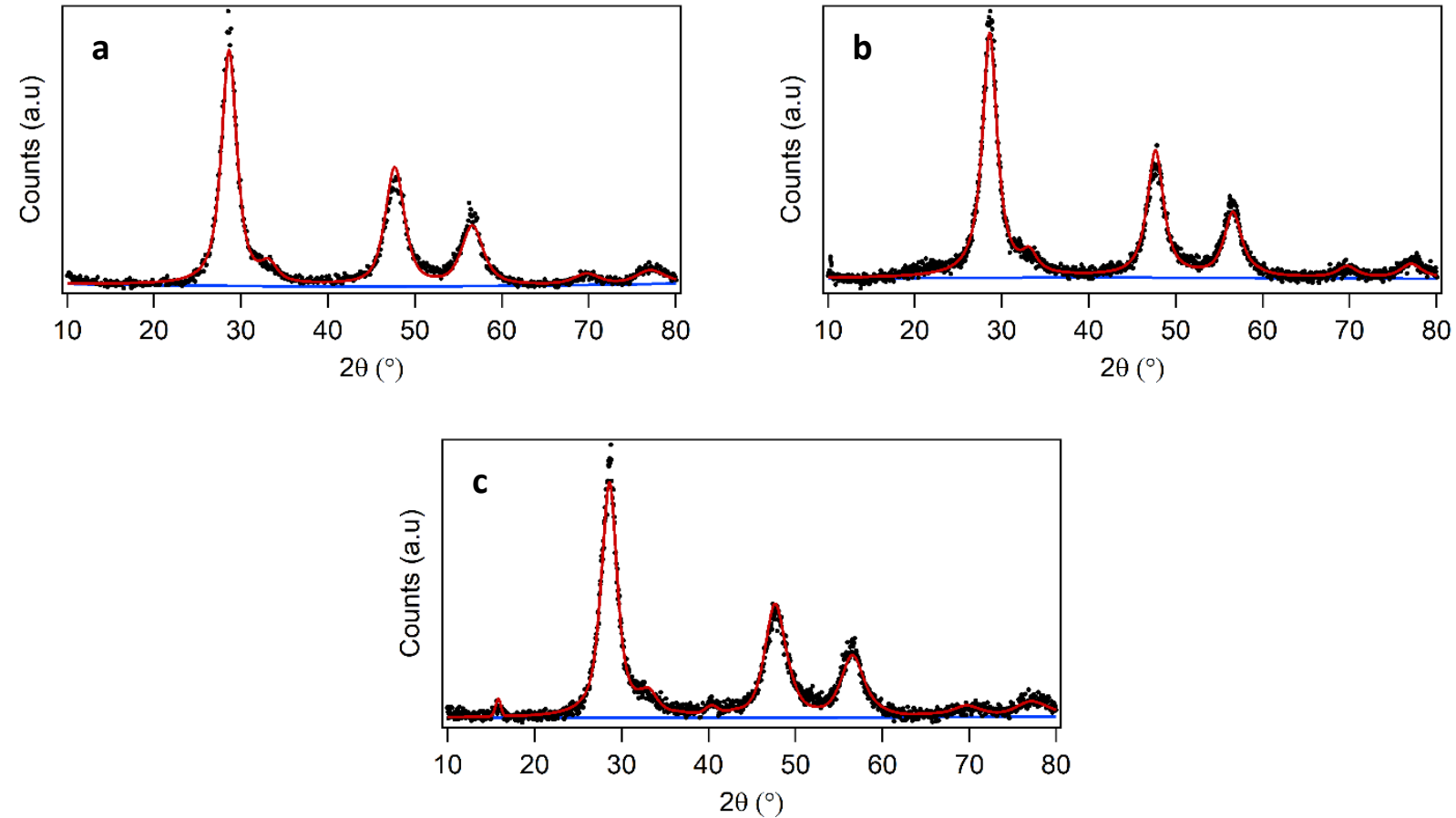

Figure S6. Fitting (red line) of diffractograms of Nd-doped ZnS samples. Doping percentages: 0.1 at $\%$ (a), 1 at $\%$ (b), 5 at $\%$ (c). Blue line: background.

Table S4. Refined parameters obtained from fitting of diffractogram of Nd-doped ZnS

\begin{tabular}{ccccccccc}
\hline Sample & Crystallite size (nm) & R.m.s. microstrain (\%) & $a(\AA)$ & $R_{\exp }(\%)$ & $\mathrm{R}_{\mathrm{wp}}(\%)$ & $\begin{array}{c}\chi^{2} \\
\mathrm{Nd}(\mathrm{OH})_{3} \\
\mathrm{wt} \%\end{array}$ \\
\hline ZnS:Nd 0.1 at\% & $5.7 \pm 0.1$ & $1.1 \pm 0.1$ & $5.39 \pm 0.01$ & 10.06 & 14.21 & 2.00 & - \\
\hline ZnS:Nd 1 at\% & $5.3 \pm 0.1$ & $0.5 \pm 0.1$ & $5.39 \pm 0.01$ & 9.75 & 12.76 & 1.71 & - \\
\hline ZnS:Nd 5 at\% & $5.5 \pm 0.1$ & $1.2 \pm 0.1$ & $5.40 \pm 0.01$ & 9.74 & 12.52 & 1.65 & $3.6 \pm 0.2$ \\
\hline
\end{tabular}
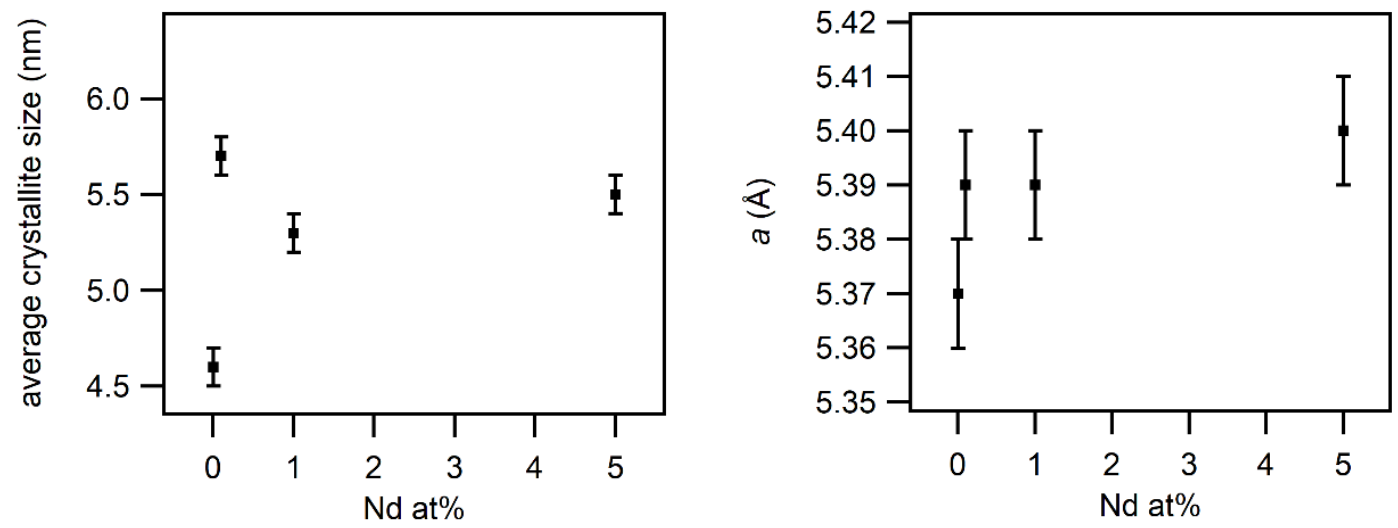

Figure S7. Average crystallite size (left) and lattice constant $a$ (right) obtained from fitting of diffractograms of Nddoped $\mathrm{ZnS}$ as a function of $\mathrm{Nd}$ atomic percentage. 
Sm-doped ZnS
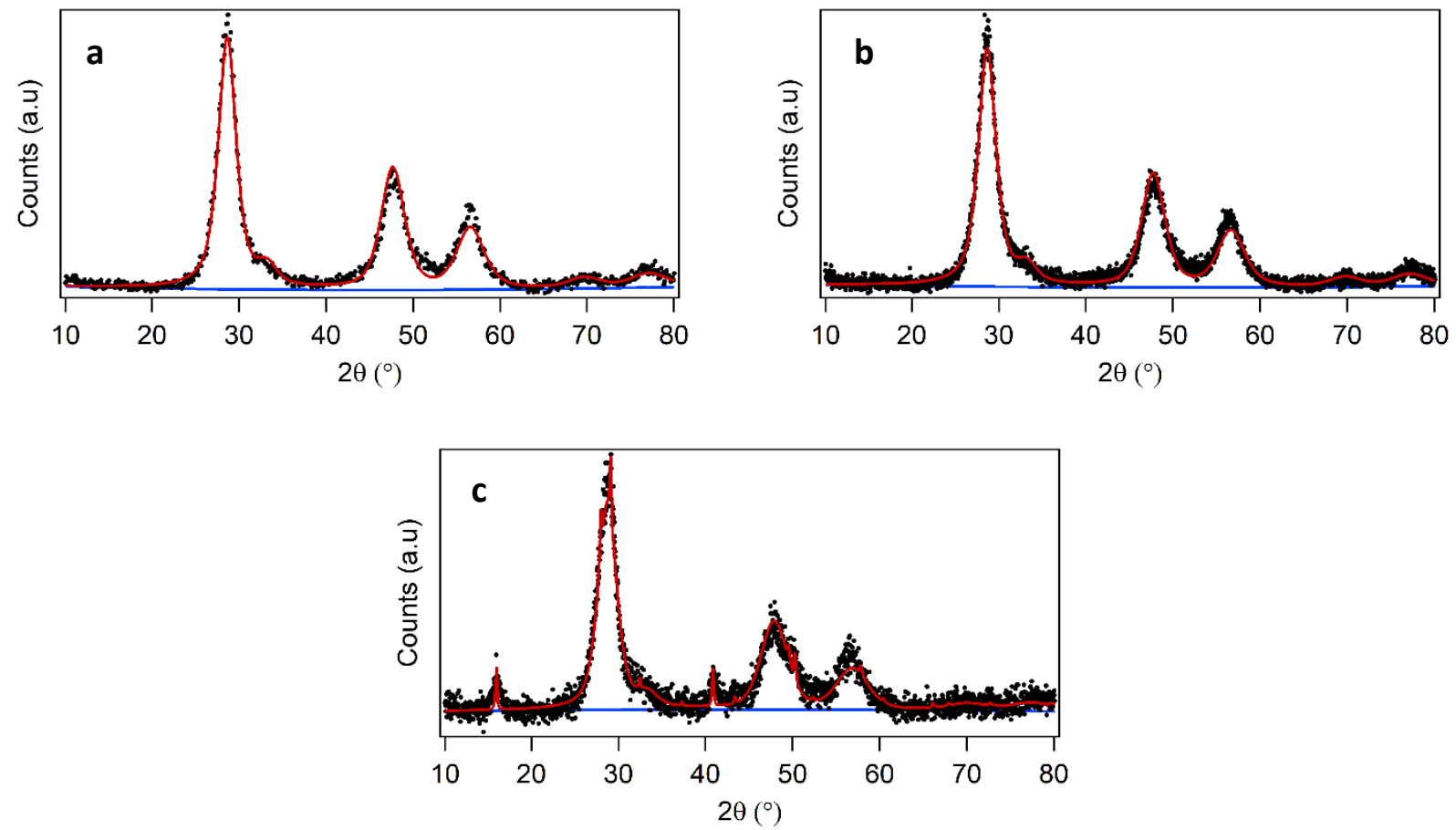

Figure S8. Fitting (red line) of diffractograms of Sm-doped ZnS samples Doping percentages: 0.1 at $\%$ (a), 1 at $\%$ (b), 5 at $\%$ (c). Blue line: background.

Table S5. Refined parameters obtained from fitting of diffractogram of Sm-doped ZnS.

\begin{tabular}{cccccccc}
\hline Sample & Crystallite size (nm) & R.m.s. microstrain (\%) & a $(\AA)$ & $\begin{array}{c}\text { Rexp } \\
(\%)\end{array}$ & $\begin{array}{c}\text { Rwp } \\
(\%)\end{array}$ & $\begin{array}{c}\chi^{2} \\
\text { sm(OH)3 } \\
\text { wt\% }\end{array}$ \\
\hline ZnS:Sm 0.1 at\% & $5.4 \pm 0.2$ & $1.6 \pm 0.1$ & $5.40 \pm 0.01$ & 10.14 & 14.89 & 2.20 & - \\
\hline ZnS:Sm 1 at\% & $5.4 \pm 0.1$ & $1.6 \pm 0.1$ & $5.39 \pm 0.01$ & 11.73 & 15.22 & 1.68 & - \\
\hline ZnS:Sm 5 at\% & $6.2 \pm 0.2$ & $2.1 \pm 0.1$ & $5.37 \pm 0.01$ & 12.17 & 14.38 & 1.40 & $3.2 \pm 0.2$ \\
\hline
\end{tabular}
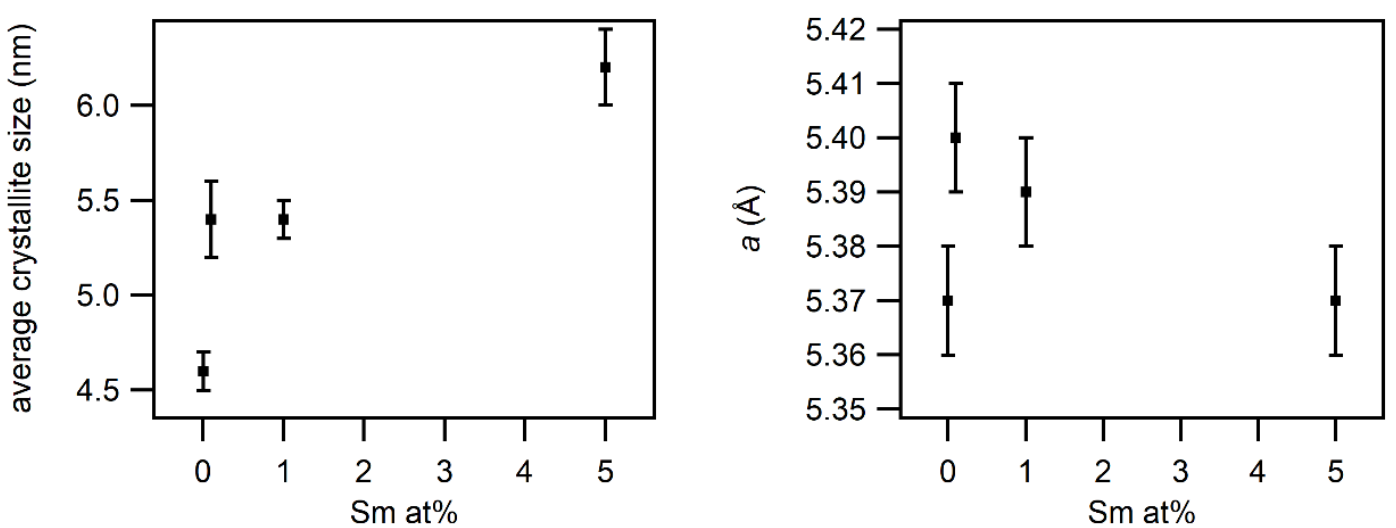

Figure S9. Average crystallite size (left) and lattice constant $a$ (right) obtained from fitting of diffractograms of Smdoped $\mathrm{ZnS}$ as a function of $\mathrm{Sm}$ atomic percentage. 
Yb-doped ZnS
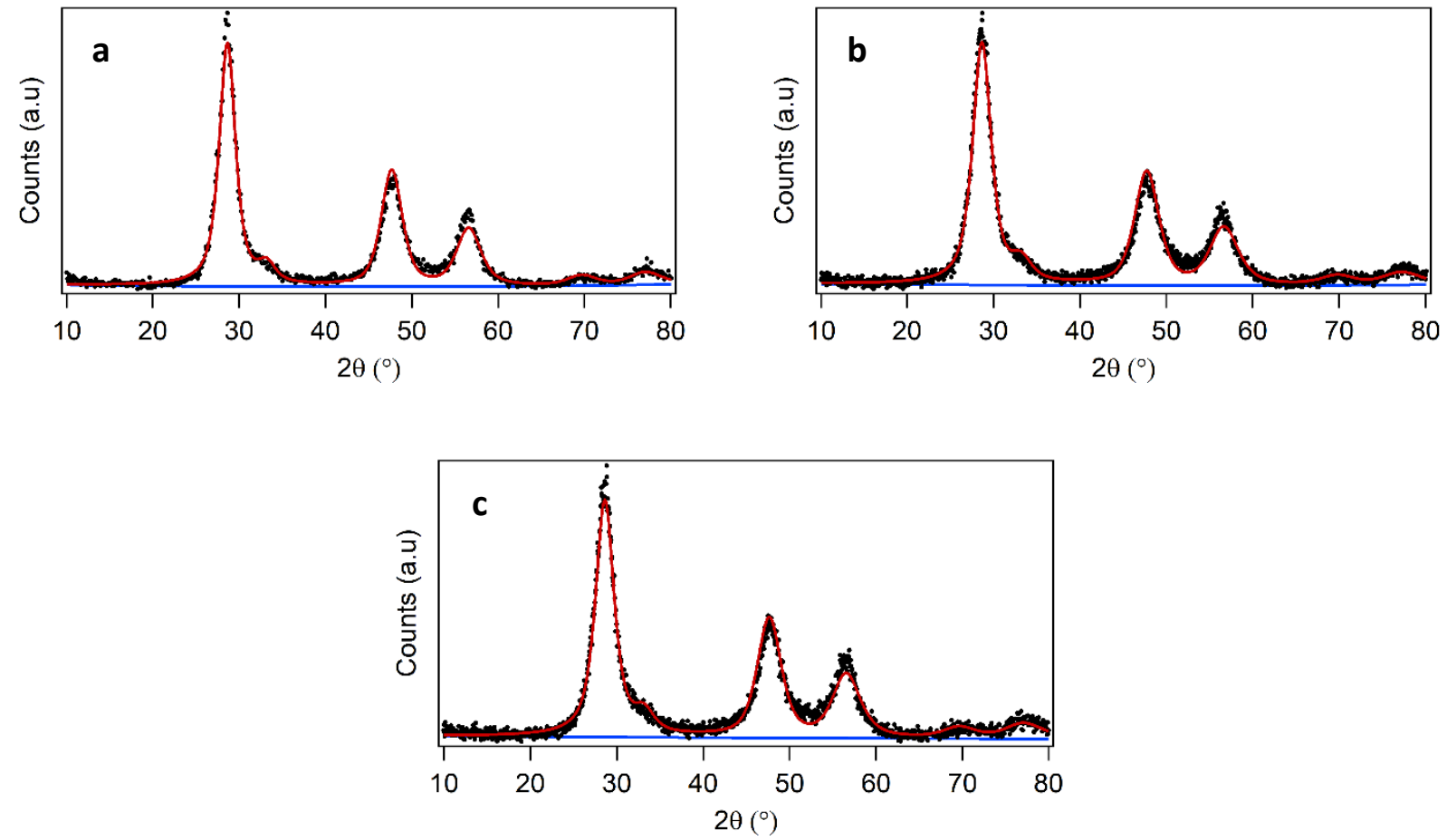

Figure S10. Fitting (red line) of diffractograms of Yb-doped ZnS samples. Doping percentages: 0.1 at\% (a), 1 at $\%$ (b), 5 at $\%$ (c). Blue line: background.

Table S6. Refined parameters obtained from fitting of diffractogram of Yb-doped ZnS.

\begin{tabular}{ccccccc}
\hline Sample & Crystallite size $(\mathrm{nm})$ & R.m.s. microstrain $(\%)$ & $a(\AA)$ & $\mathrm{R}_{\exp }(\%)$ & $\mathrm{R}_{\mathrm{wp}}(\%)$ & $\chi^{2}$ \\
\hline $\mathrm{ZnS}: \mathrm{Yb} \mathrm{0.1} \mathrm{at \%}$ & $5.7 \pm 0.2$ & $1.4 \pm 0.1$ & $5.40 \pm 0.01$ & 9.41 & 14.34 & 2.32 \\
\hline $\mathrm{ZnS}: \mathrm{Yb} \mathrm{1} \mathrm{at \%}$ & $5.0 \pm 0.1$ & $1.6 \pm 0.1$ & $5.39 \pm 0.01$ & 9.56 & 13.19 & 1.90 \\
\hline $\mathrm{ZnS}: \mathrm{Yb} \mathrm{5}$ at\% & $4.9 \pm 0.1$ & $1.5 \pm 0.1$ & $5.40 \pm 0.01$ & 10.06 & 14.01 & 1.94 \\
\hline
\end{tabular}
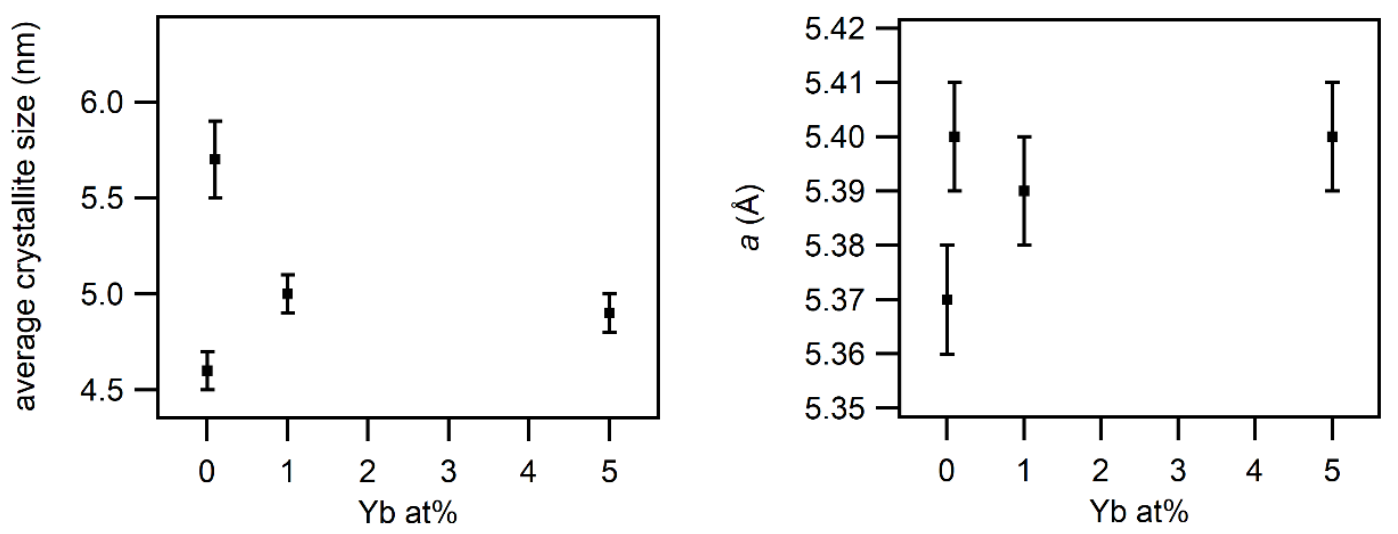

Figure S11. Average crystallite size (left) and lattice constant $a$ (right) obtained from fitting of diffractograms of $\mathrm{Yb}$-doped $\mathrm{ZnS}$ as a function of $\mathrm{Yb}$ atomic percentage. 

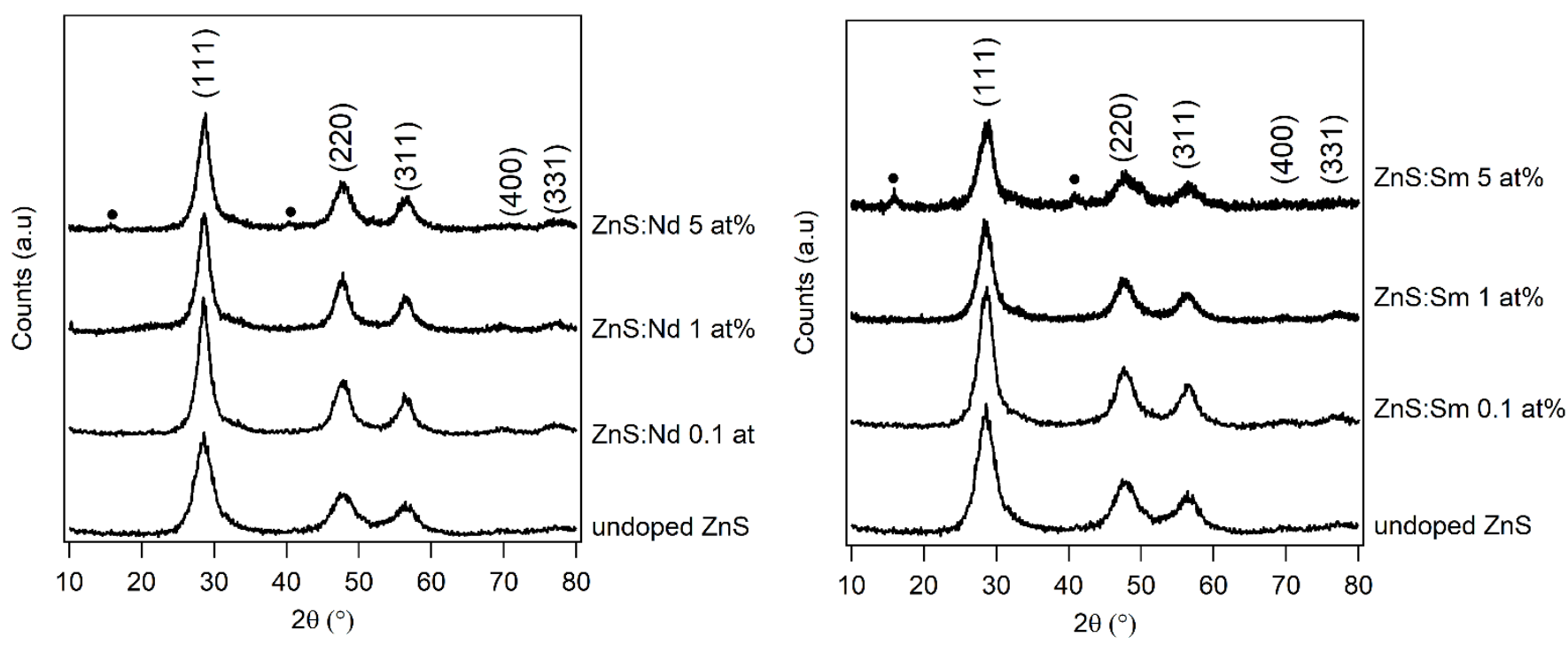

Figure S12. Comparison of diffractograms of undoped and Nd-doped (left), Sm-doped (right) at different atomic percentages. Ln(OH) 3 reflections are highlighted by a $\bullet$.

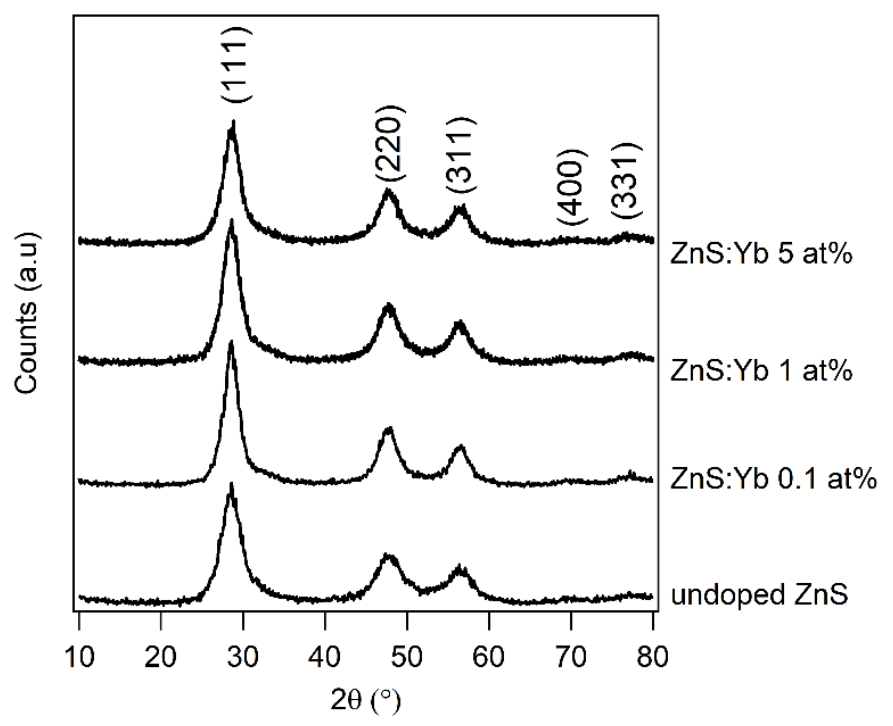

Figure S13. Comparison of diffractograms of undoped and Yb-doped ZnS samples at different atomic percentages. 


\section{Transmission Electron Microscopy and High-Resolution Transmission Electron Microscopy.}

Undoped ZnS
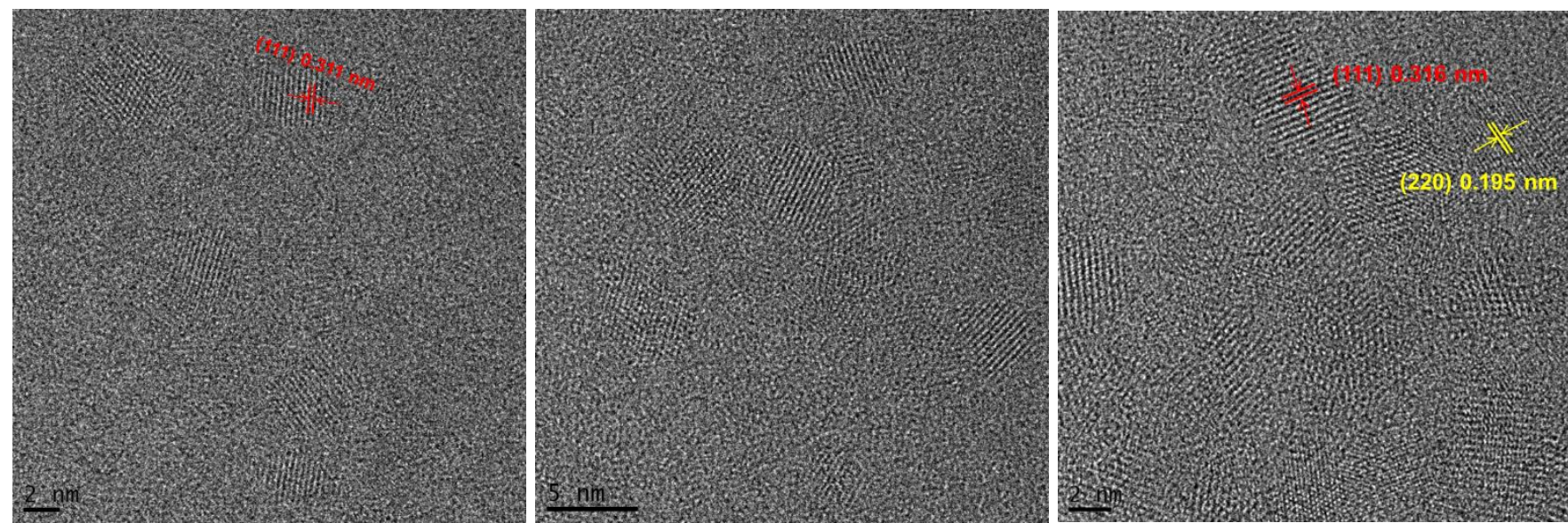

Figure S14. HRTEM micrographs of undoped zinc sulfide nanoparticles.

$\mathrm{ZnS}: \mathrm{Mn} 5$ at $\%$
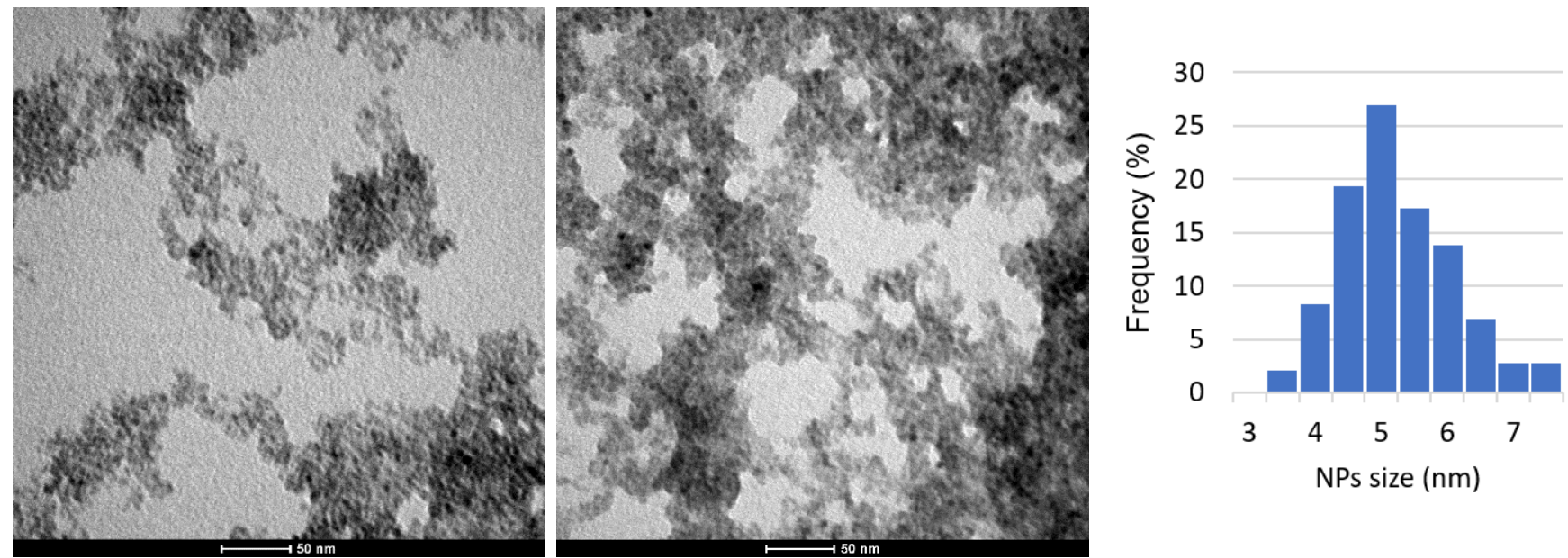

Figure S15. TEM micrographs of 5 at\% Mn-doped zinc sulfide nanoparticles (left and center) and size distribution histogram (right). 

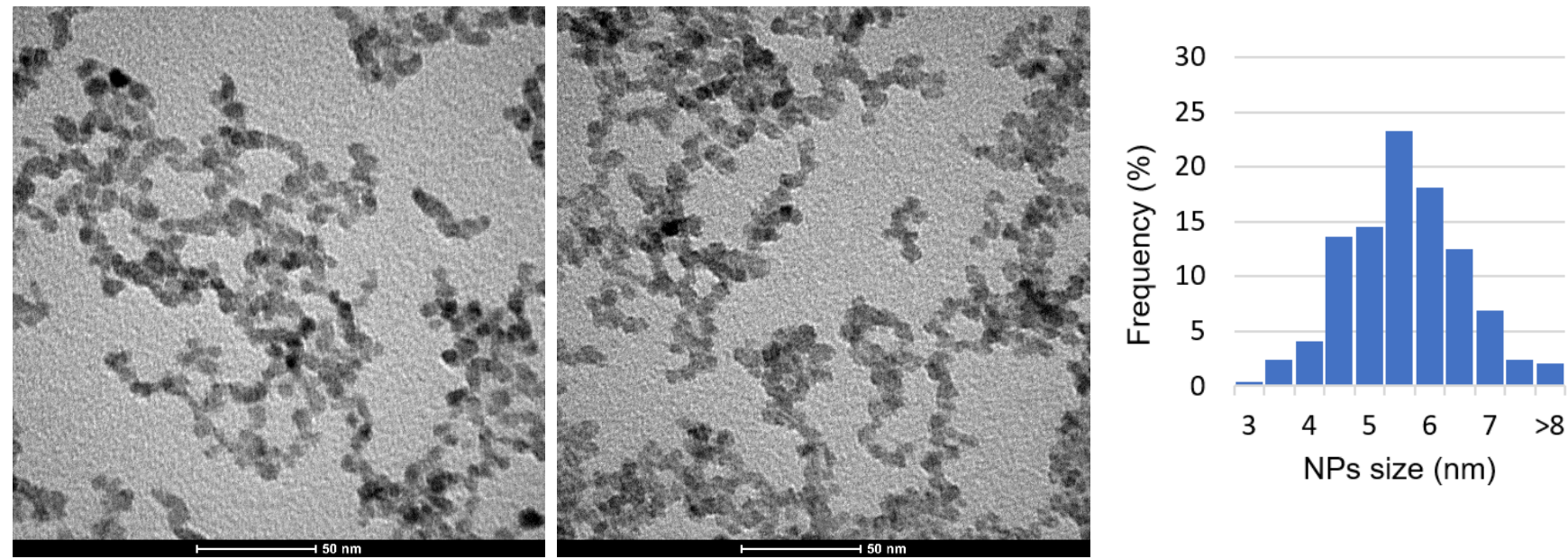

Figure S16. TEM micrographs of 5 at\% Eu-doped zinc sulfide nanoparticles (left and center) and size distribution histogram (right).
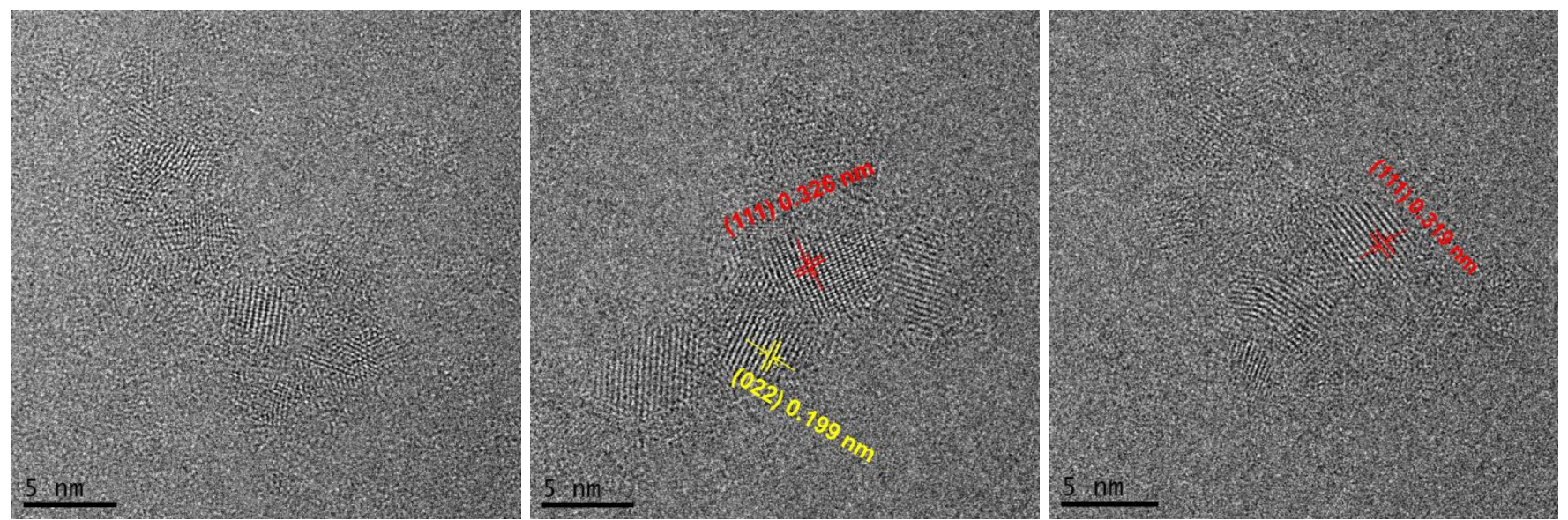

Figure S17. HRTEM micrographs of 5 at\% Eu-doped zinc sulfide nanoparticles. 

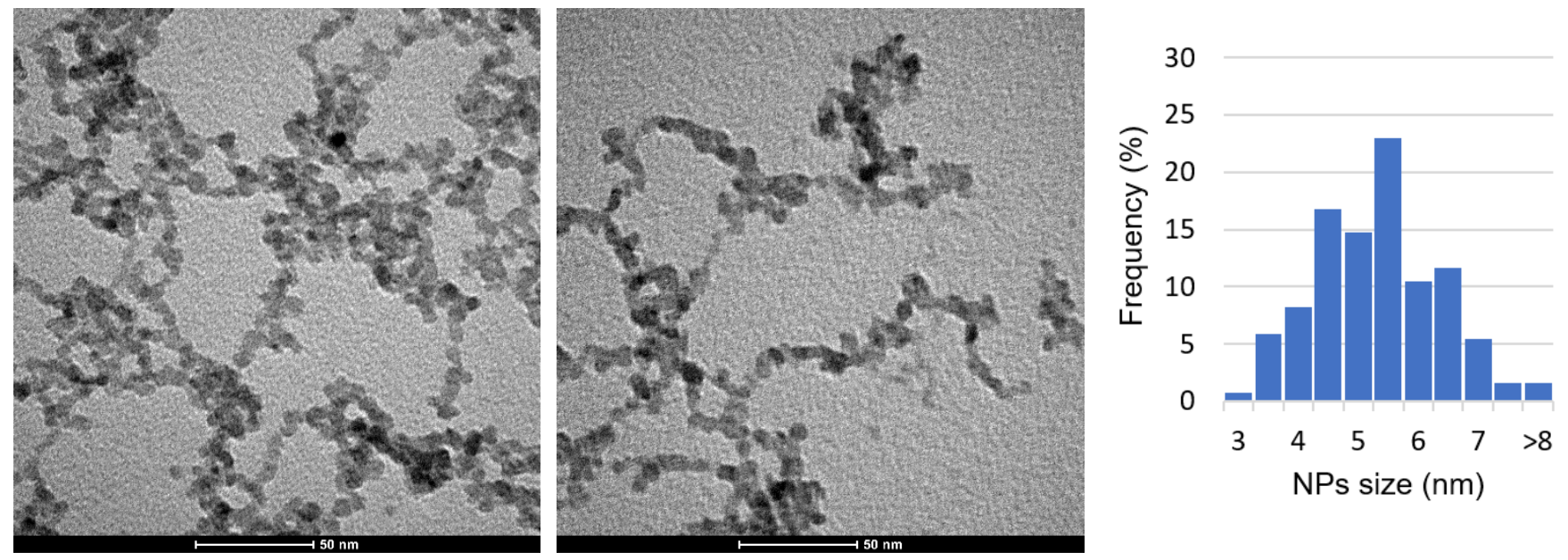

Figure S18. TEM micrographs of 5 at\% Nd-doped zinc sulfide nanoparticles (left and center) and size distribution histogram (right).

$\mathrm{ZnS}: \mathrm{Sm} 5$ at\%
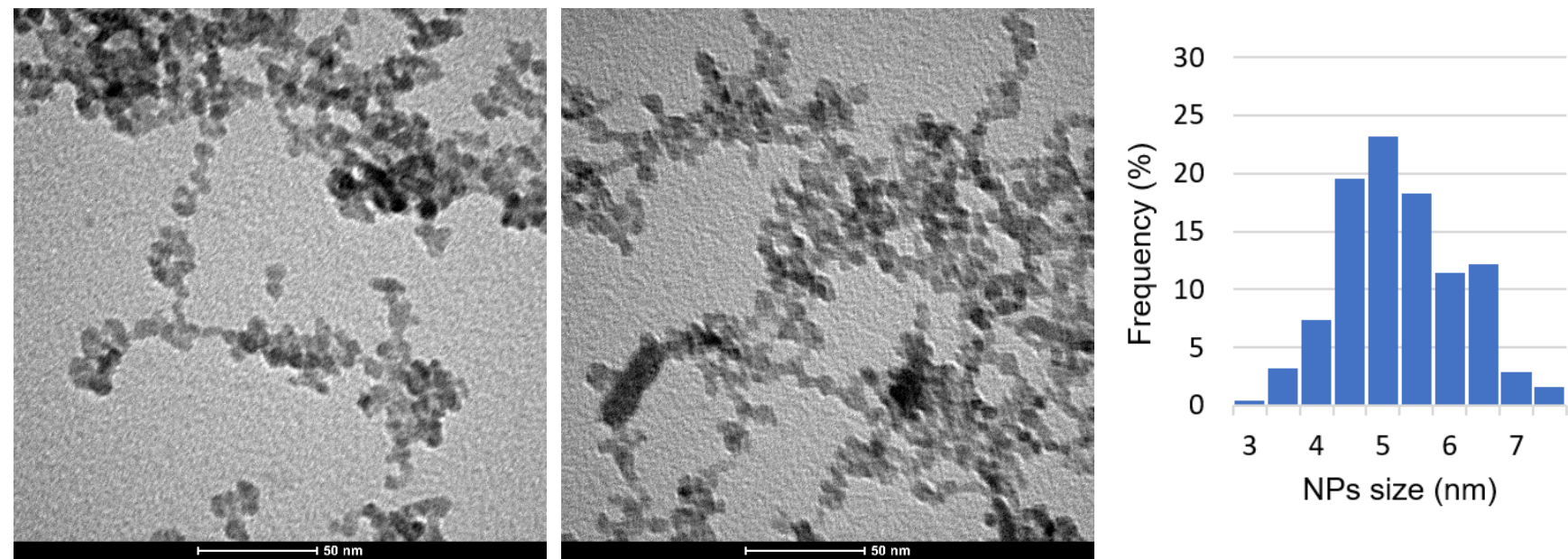

Figure S19. TEM micrographs of 5 at\% Sm-doped zinc sulfide nanoparticles (left and center) and size distribution histogram (right). 

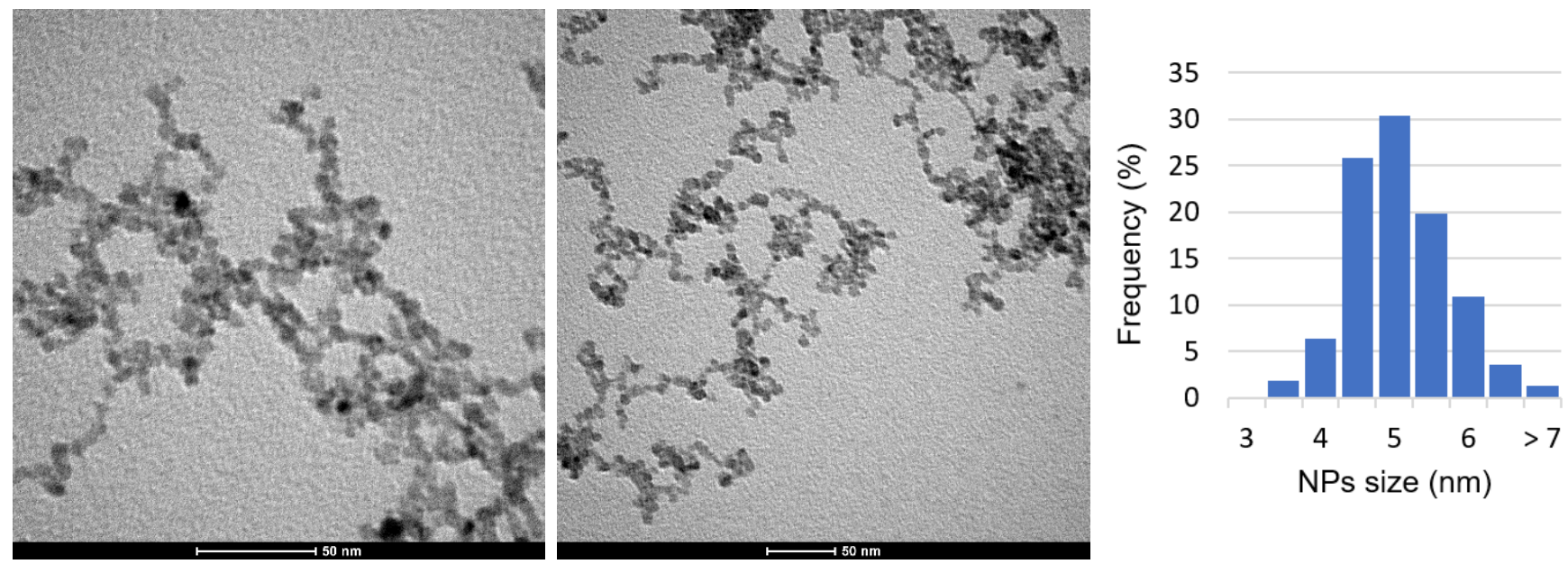

Figure S20. TEM micrographs of 5 at\% Yb-doped zinc sulfide nanoparticles (left and center) and size distribution histogram (right). 


\section{X-ray Photoelectron Spectroscopy.}

Undoped ZnS
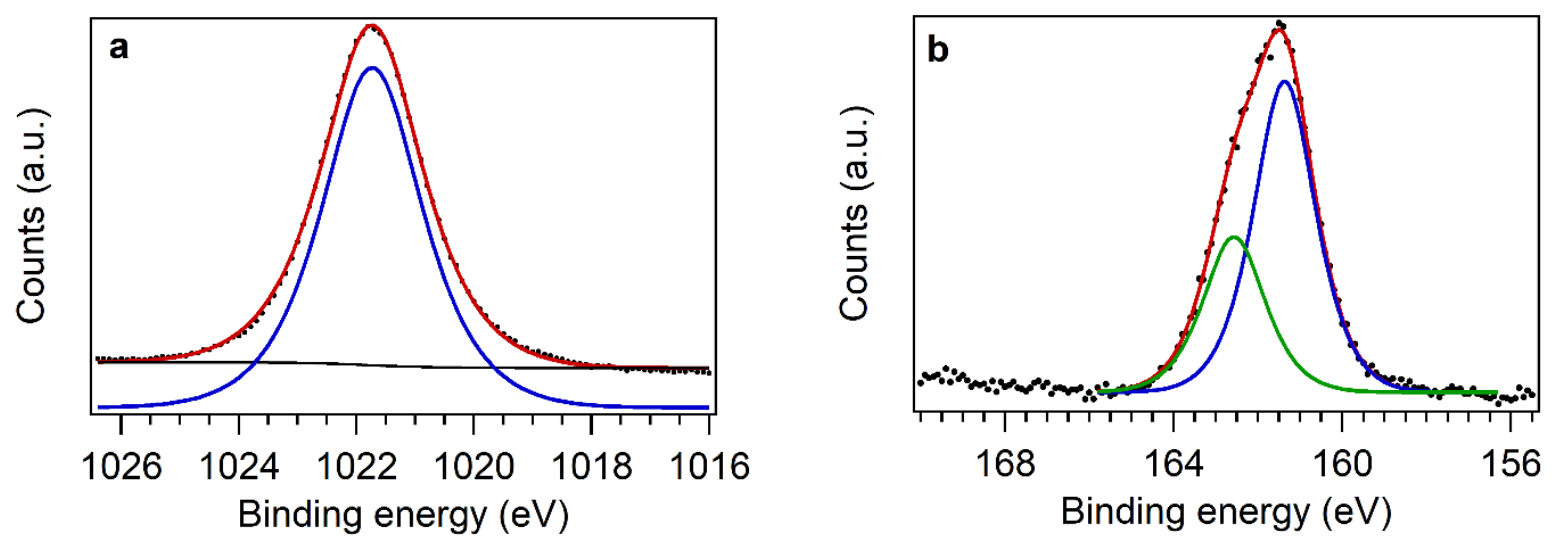

Figure S21. Fittings (red lines) of Zn2p $3 / 2$ XPS peak (a) (blue line: photoemission peak) and S2p XPS peak (b) (blue line: S2p $3 / 2$ component; green line: S2 $\mathrm{p}_{1 / 2}$ component, separated for clarity) of undoped $\mathrm{ZnS}$. BE values are corrected for charge effects.
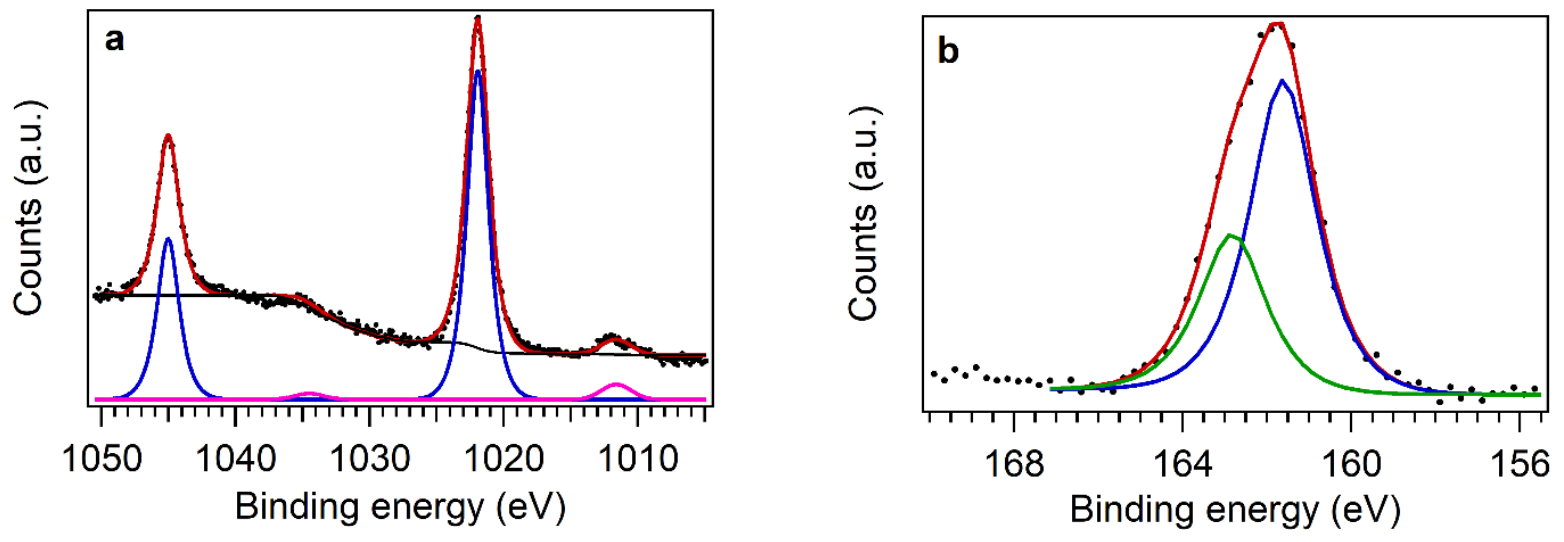

Figure S22. Fittings (red lines) of Zn2p XPS peaks (a) (blue line: photoemission peaks; pink line: shake up satellite peaks) and of S2p XPS peak (b) (blue line: S2p $3 / 2$ component; green line: S2 $\mathrm{p}_{1 / 2}$ component, separated for clarity) of ZnS:Mn 5 at $\%$. BE values are corrected for charge effects. 

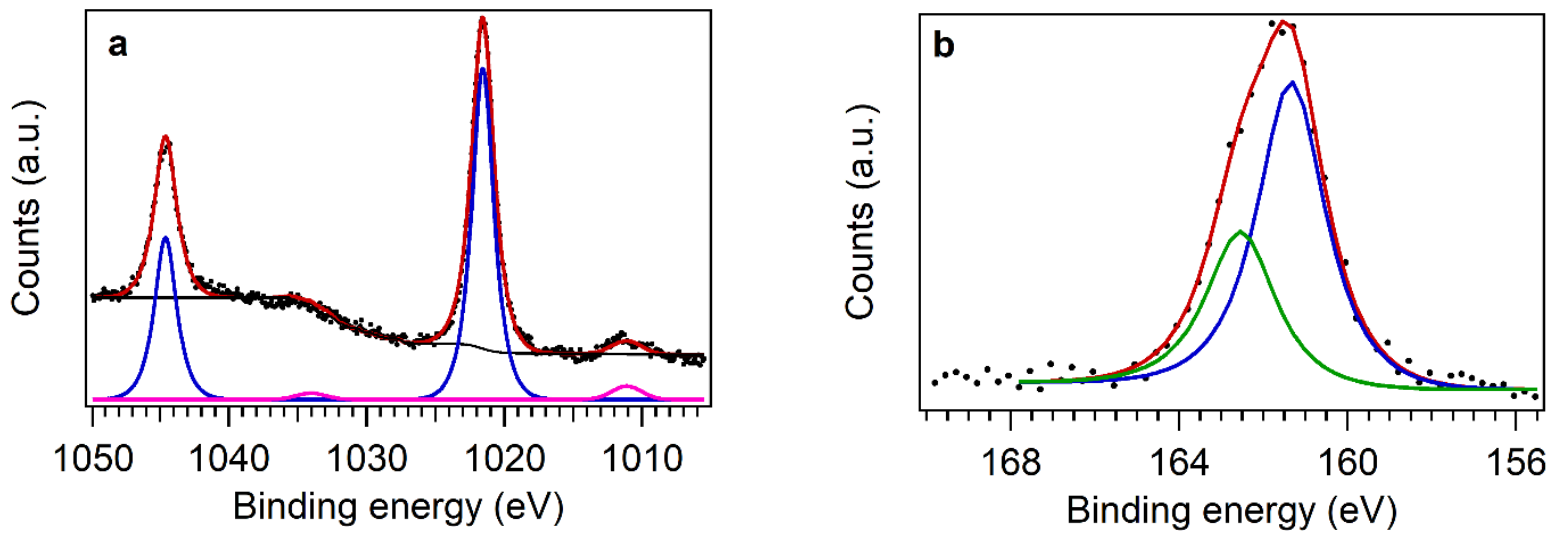

Figure S23. Fittings (red lines) of Zn2p XPS peaks (a) (blue line: photoemission peaks; pink line: shake up satellite peaks) and of S2p XPS peak (b) (blue line: S2p3/2 component; green line: S2p $1 / 2$ component, separated for clarity) of ZnS:Eu 5 at\%. BE values are corrected for charge effects.

\section{$\mathrm{ZnS}: \mathrm{Sm} 5$ at $\%$}
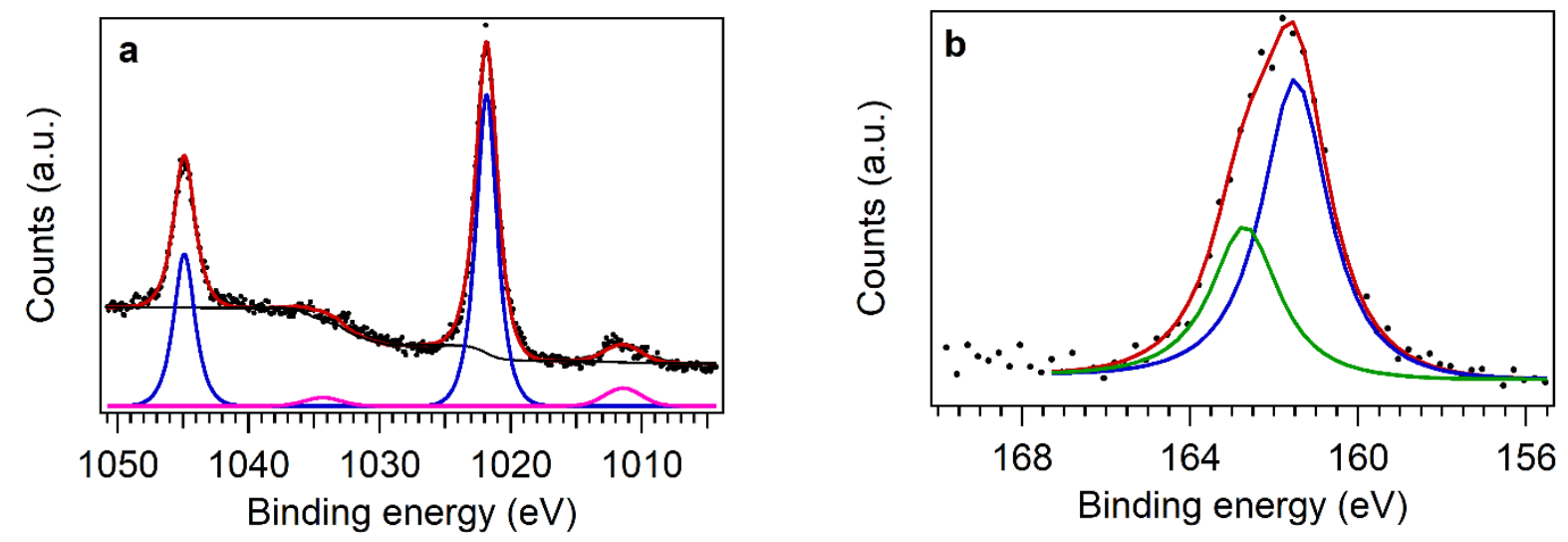

Figure S24. Fittings (red lines) of Zn2p XPS peaks (a) (blue line: photoemission peaks; pink line: shake up satellite peaks) and of S2p XPS peak (b) (blue line: S2 $p_{3 / 2}$ component; green line: S2 $p_{1 / 2}$ component, separated for clarity) of $\mathrm{ZnS:Sm} 5$ at $\%$. BE values are corrected for charge effects. 

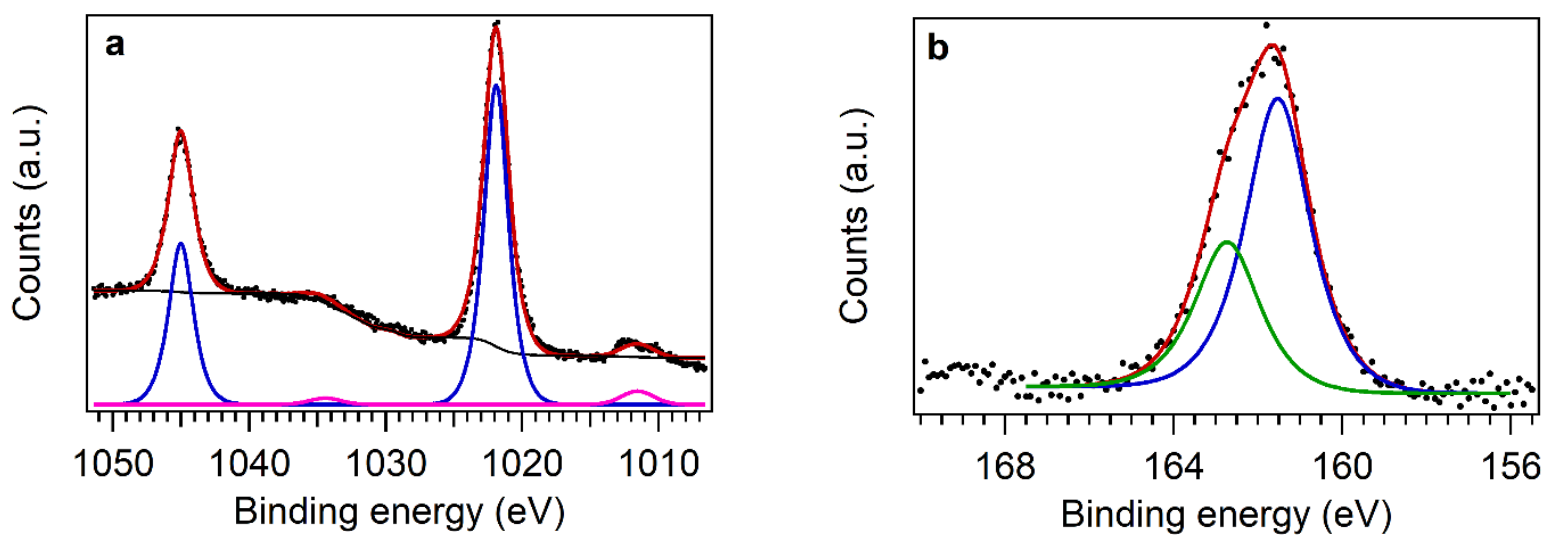

Figure S25. Fitting (red lines) of Zn2p XPS peaks (a) (blue line: photoemission peaks; pink line: shake up satellite peaks) and of S2p XPS peak (b) (blue line: S2 $p_{3 / 2}$ component; green line: S2 $\mathrm{p}_{1 / 2}$ component, separated for clarity) of $\mathrm{ZnS}: \mathrm{Yb} 5$ at $\%$. BE values are corrected for charge effects

Table S7 - Surface atomic composition (\%) obtained from semi-quantitative analysis of ZnS2p3/2 and S2p XPS peaks.

\begin{tabular}{clc}
\hline Sample & Zn & S \\
\hline Undoped ZnS & 1 & 0.8 \\
\hline ZnS:Mn 5 at\% & 1 & 0.8 \\
\hline ZnS:Eu 5 at\% & 1 & 0.7 \\
\hline ZnS:Nd 5 at\% & 1 & 0.8 \\
\hline ZnS:Sm 5 at\% & 1 & 0.7 \\
\hline ZnS:Yb 5 at\% & 1 & 0.5
\end{tabular}

Table S8 - Binding energy (BE) and Auger parameter (AP) values.

\begin{tabular}{cccc}
\hline Sample & BE Zn2p3/2 (eV) & AP (eV) & BE S2p (eV) \\
\hline ZnS:Sm 5 at\% & 1021.6 & 2011.4 & 161.4 \\
\hline ZnS:Yb 5 at\% & 1021.9 & 2011.5 & 161.4
\end{tabular}


ICP-MS Analysis.

Table S9. ICP-MS measurements (error: $\pm 5 \%$ ).

\begin{tabular}{lcc}
\hline & Atomic percentage \\
& $\frac{[\mathbf{M}]}{[\mathbf{Z n}]+[\mathbf{M}]} \cdot \mathbf{1 0 0}$ \\
& Nominal & Experimental \\
& 0.1 & 0.09 \\
$\mathrm{ZnS}: \mathrm{Sm}$ & 1 & 0.90 \\
& 5 & 5.44 \\
& 0.1 & 0.08 \\
$\mathrm{ZnS}: \mathrm{Yb}$ & 1 & 0.48 \\
& 5 & 2.65 \\
\hline
\end{tabular}

S-16 
X-ray Absorption Spectroscopy.
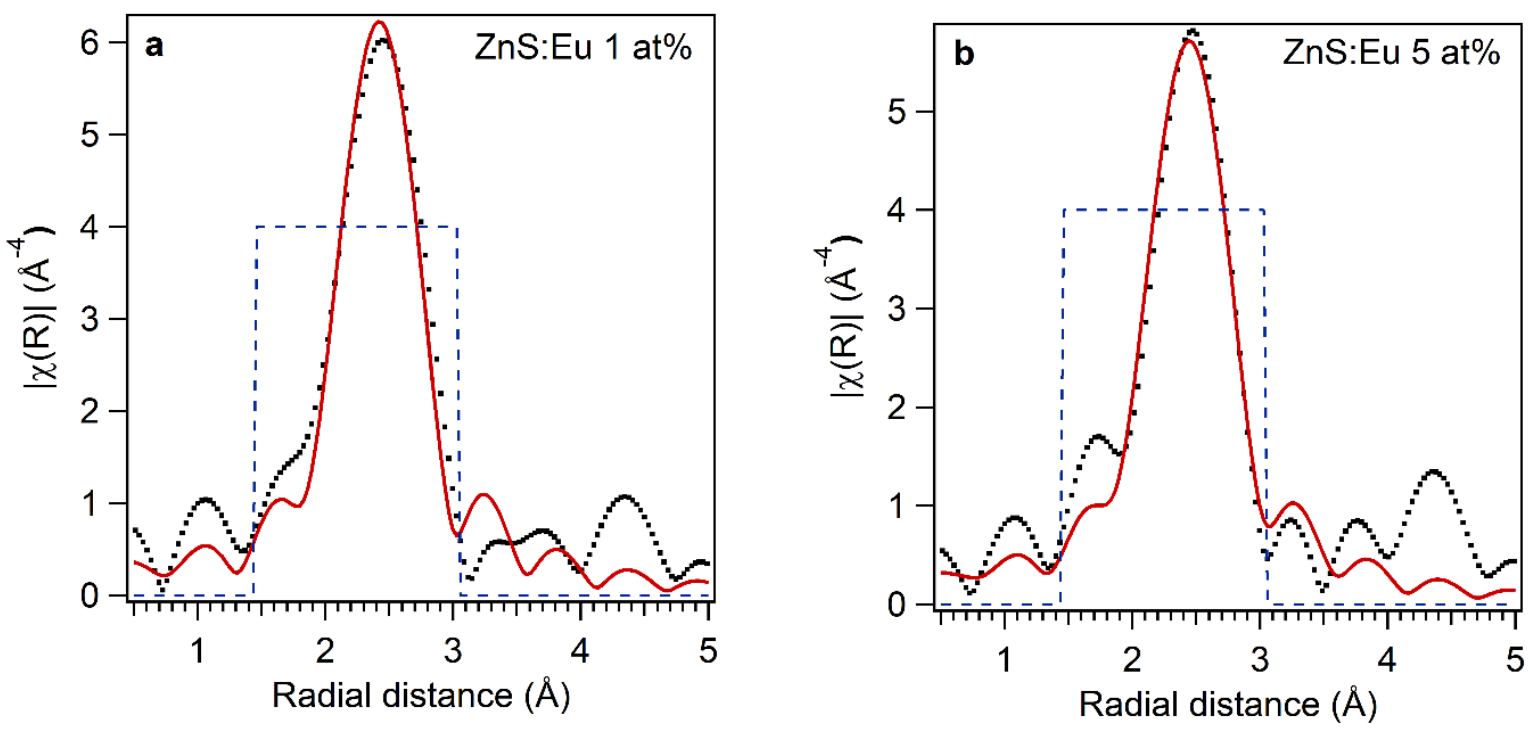

Figure S26. Fitted Fourier transformed EXAFS data of ZnS:Eu 1 at\% (a) and ZnS:Eu 5 at\% (b). Blue line: window for fitting range. Data are corrected for scattering phase-shift. 

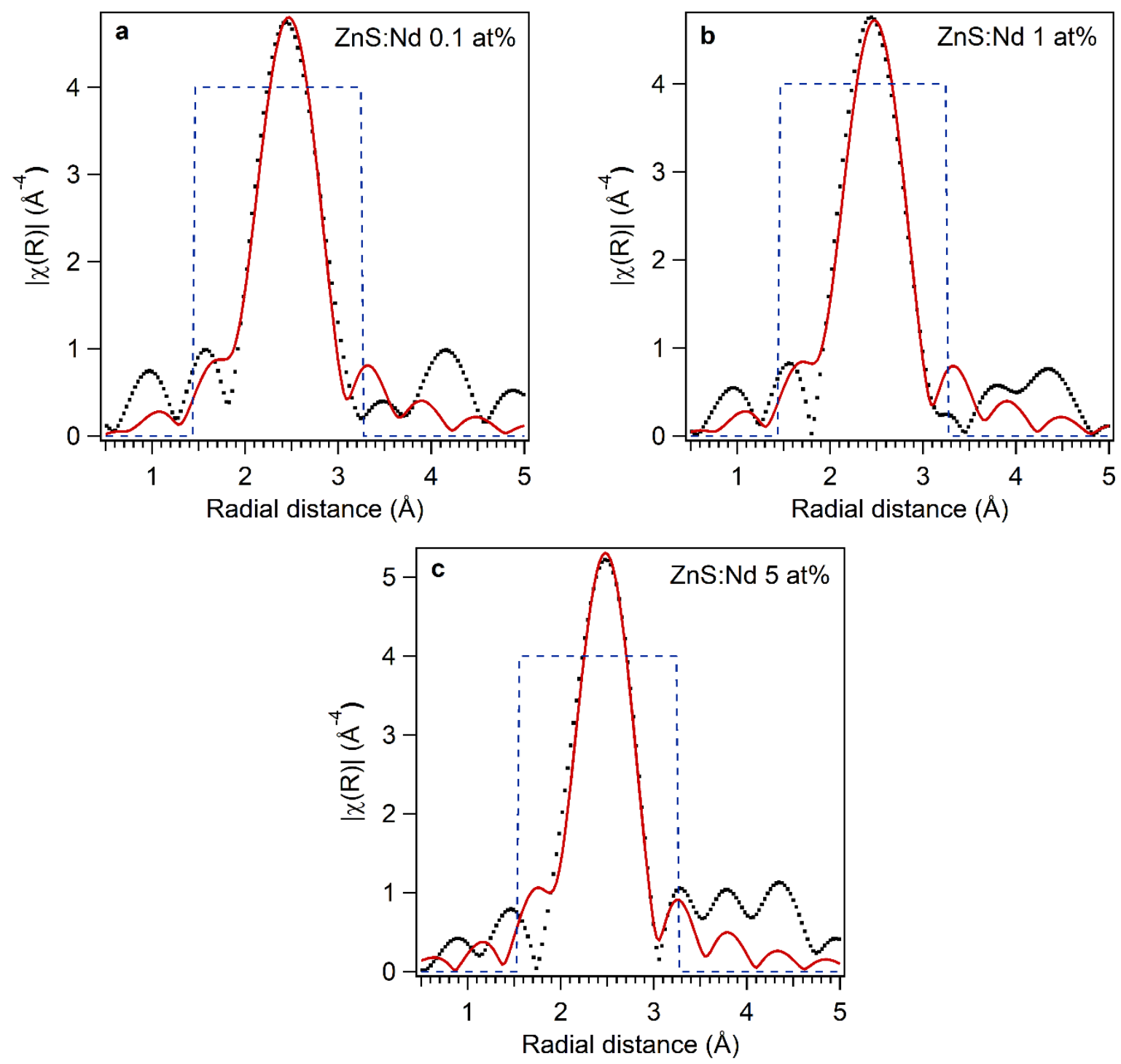

Figure S27. Fitted Fourier transformed EXAFS data of ZnS:Nd 0.1 at $\%$ (a), ZnS:Nd 1 at $\%$ (b) and ZnS:Nd 5 at\% (c). Blue line: window for fitting range. Data are corrected for scattering phase-shift. 


\section{Absorption and photoluminescence spectroscopy.}

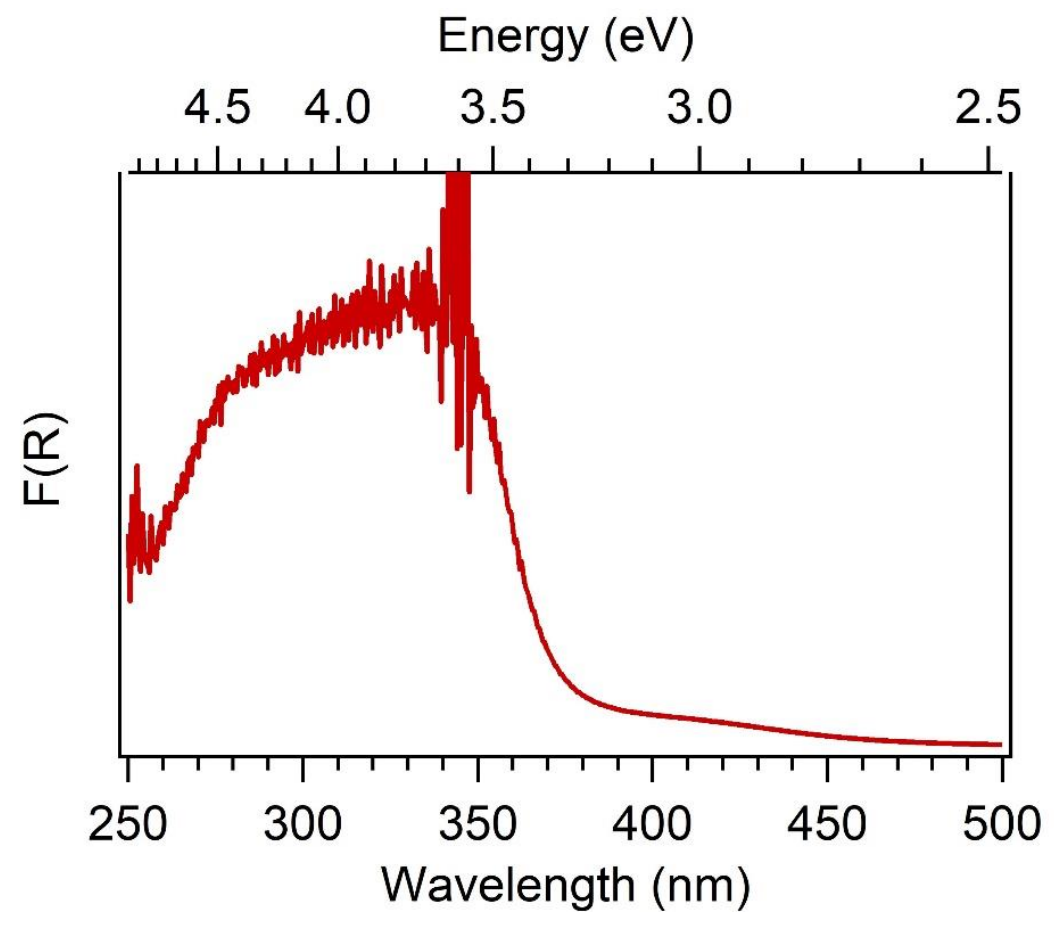

Figure S28. Diffuse reflectance spectrum of undoped ZnS sample.

The reflectance data in the 3.55-3.65 eV (350-340 nm) range are affected by some noise due to the change of the lamp of the spectrophotometer during the measurement and therefore in this part of the spectrum the signal shows relevant oscillations, as it can be noted in Figure S26. Nonetheless, an increasing behaviour of the reflectance is clearly observed from the visible region up to $330-340 \mathrm{~nm}$, followed by a decrease of the reflectance on increasing the photon energy.

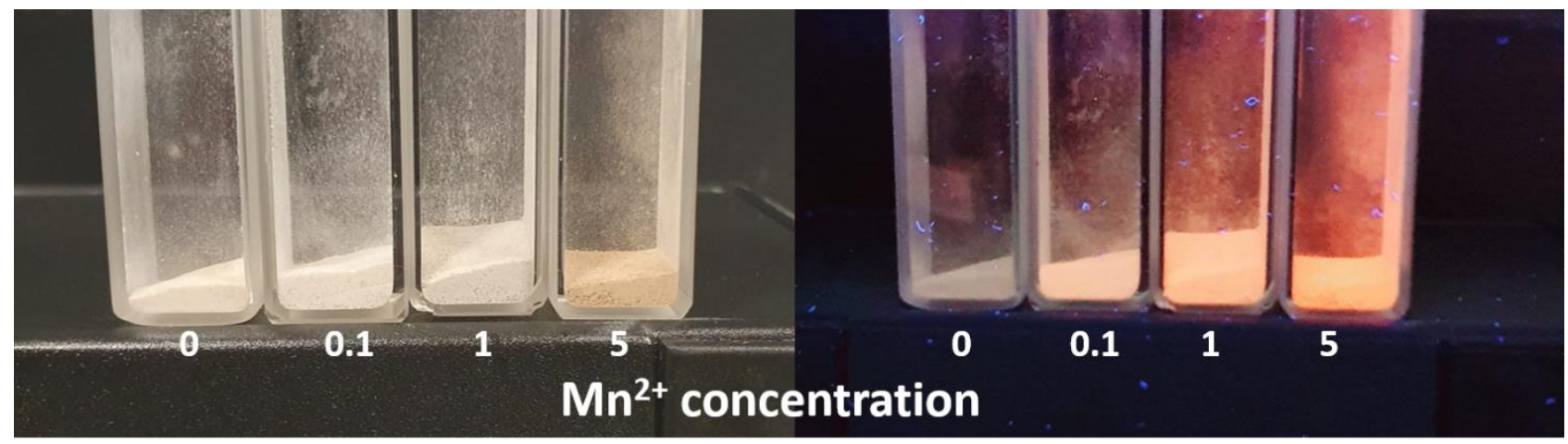

Figure S29. Picture of undoped and Mn-doped ZnS samples at different atomic concentrations, before (left) and during (right) UV illumination. 


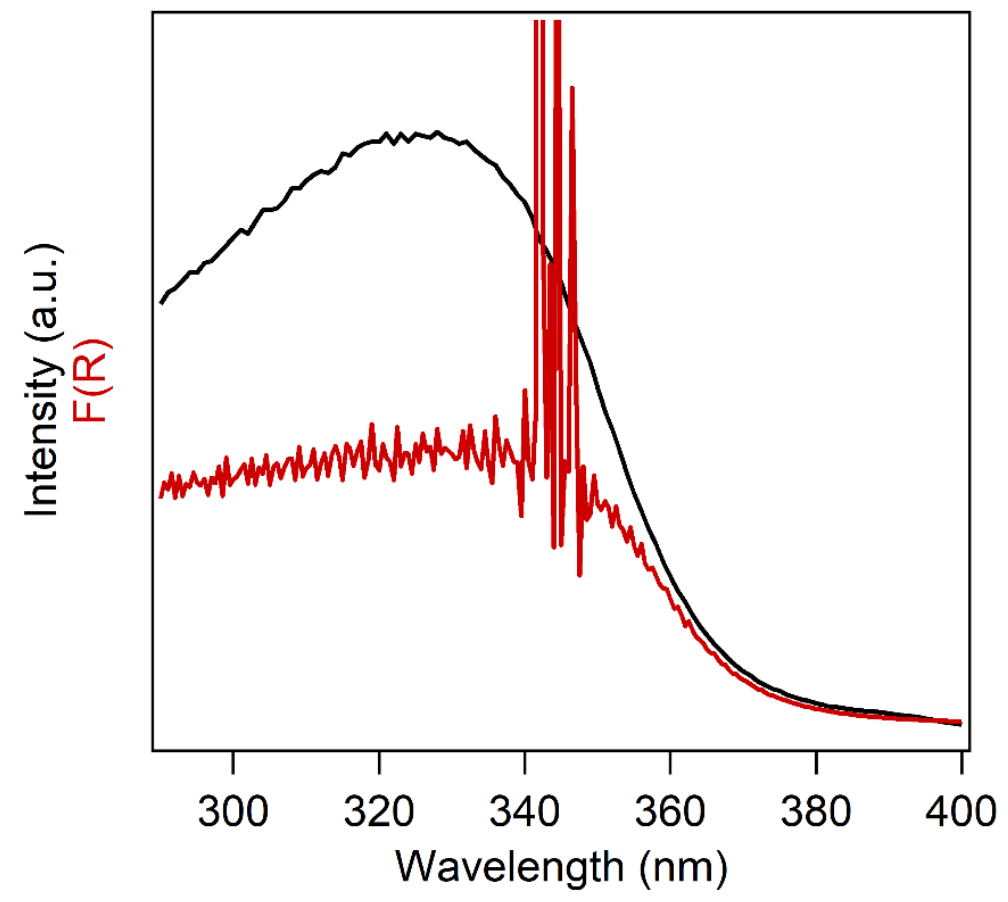

Figure S30. Comparison between absorption band of undoped ZnS (red line) and excitation band of 0.1 at $\%$ Mn-doped sample (black line, $\lambda_{\mathrm{em}}=597 \mathrm{~nm}$ ).

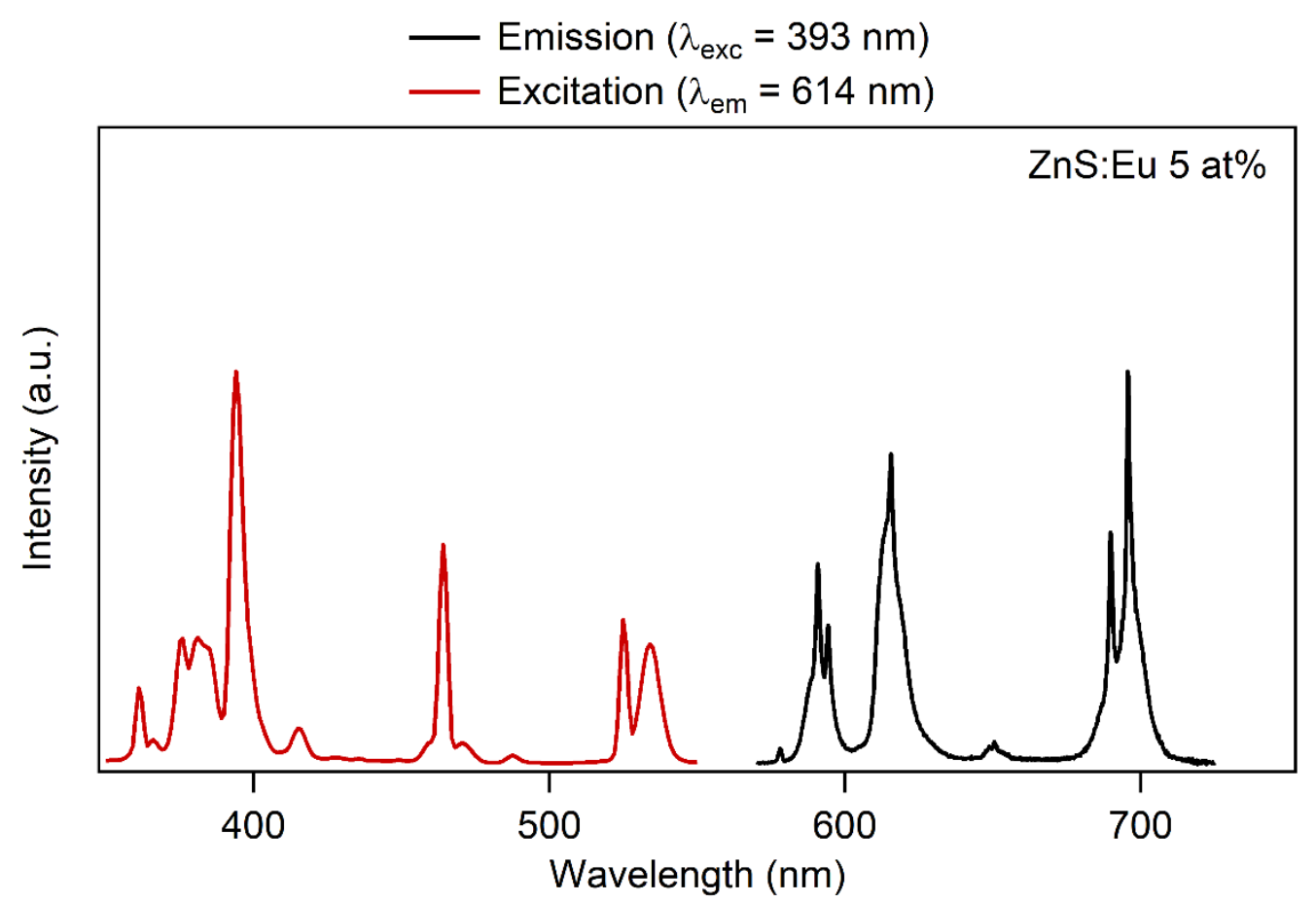

Figure S31. Emission spectrum (black line, $\lambda_{\mathrm{exc}}=393 \mathrm{~nm}$ ) and excitation spectrum (red line, $\lambda_{\mathrm{em}}=614 \mathrm{~nm}$ ) of and $\mathrm{ZnS}: \mathrm{Eu} 5 \mathrm{at} \%$. 